\title{
Genome-level diversification of eight ancient tea populations in the Guizhou and Yunnan regions identifies candidate genes for core agronomic traits
}

\author{
Litang Lu ${ }^{1,2}$, Hufang Chen ${ }^{1,2}$, Xiaojing Wang ${ }^{1}$, Yichen Zhao ${ }^{1,2}$, Xinzhuan Yao ${ }^{1}$, Biao Xiong ${ }^{1}$, Yanli Deng ${ }^{1}$ and \\ Degang Zhao $2,3 凶$
}

\begin{abstract}
The ancient tea plant, as a precious natural resource and source of tea plant genetic diversity, is of great value for studying the evolutionary mechanism, diversification, and domestication of plants. The overall genetic diversity among ancient tea plants and the genetic changes that occurred during natural selection remain poorly understood. Here, we report the genome resequencing of eight different groups consisting of 120 ancient tea plants: six groups from Guizhou Province and two groups from Yunnan Province. Based on the 8,082,370 identified high-quality SNPs, we constructed phylogenetic relationships, assessed population structure, and performed genome-wide association studies (GWAS). Our phylogenetic analysis showed that the 120 ancient tea plants were mainly clustered into three groups and five single branches, which is consistent with the results of principal component analysis (PCA). Ancient tea plants were further divided into seven subpopulations based on genetic structure analysis. Moreover, it was found that the variation in ancient tea plants was not reduced by pressure from the external natural environment or artificial breeding (nonsynonymous/synonymous = 1.05). By integrating GWAS, selection signals, and gene function prediction, four candidate genes were significantly associated with three leaf traits, and two candidate genes were significantly associated with plant type. These candidate genes can be used for further functional characterization and genetic improvement of tea plants.
\end{abstract}

\section{Introduction}

The leaves of the tea plant Camellia sinensis (L.) O. Kuntze var. sinensis $(2 n=2 x=30)$ are used to produce different kinds of tea, making tea an important economic crop worldwide. With its attractive aroma and pleasant taste $^{1,2}$, tea is the most popular nonalcoholic caffeine-

\footnotetext{
Correspondence: Degang Zhao (dgzhao@gzu.edu.cn)

${ }^{1}$ College of Tea Science, Guizhou University, Guiyang 550025, People's

Republic of China

${ }^{2}$ College of Life Sciences and The Key Laboratory of Plant Resources

Conservation and Germplasm Innovation in the Mountainous Region (Ministry

of Education), Institute of Agro-Bioengineering, Guizhou University, Guiyang

550025, People's Republic of China

Full list of author information is available at the end of the article

These authors contributed equally: Litang Lu, Hufang Chen, Xiaojing Wang
}

containing beverage in the world and is consumed daily by more than three billion people across 160 countries. Tea beverages are rich in beneficial compounds, such as polyphenols, caffeine, theanine, vitamins, polysaccharides, volatile oils, and minerals, which have been shown to reduce the risk of developing cancer and cardiovascular, cerebrovascular, and nervous system diseases ${ }^{3-7}$. The Camellia species encompasses highly diverse crops that produce secondary metabolites in the buds and young leaves, which were targets of selection during the process of domestication. Thus, the leaf inclusions and morphological characteristics of tea plants can be used as an indicator of the selection process in tea plant breeding. At present, cultivated tea plants include two main varieties:

\section{(c) The Author(s) 2021}

(c) (i) Open Access This article is licensed under a Creative Commons Attribution 4.0 International License, which permits use, sharing, adaptation, distribution and reproduction cc) in any medium or format, as long as you give appropriate credit to the original author(s) and the source, provide a link to the Creative Commons license, and indicate if changes were made. The images or other third party material in this article are included in the article's Creative Commons license, unless indicated otherwise in a credit line to the material. If material is not included in the article's Creative Commons license and your intended use is not permitted by statutory regulation or exceeds the permitted use, you will need to obtain permission directly from the copyright holder. To view a copy of this license, visit http://creativecommons.org/licenses/by/4.0/. 
C. sinensis var. sinensis (Chinese type tea; CSS) and C. sinensis var. assamica (Assam type tea; CSA) ${ }^{8,9}$.

Analyses of genome-wide genetic diversity and the identification of genes associated with excellent traits that contribute to domestication and improvement play an essential role in the breeding of superior varieties ${ }^{10-12}$. Genome-wide association studies (GWAS) using wholegenome resequencing identified new genes influencing agronomic traits in crop plants ${ }^{13-17}$. The release of the tea genome database laid the foundation for genome resequencing and $\mathrm{GWAS}^{18}$. Resequencing dozens of tea cultivars has allowed preliminary understanding of the genetic variation patterns during tea plant domestication and varietal improvement; however, the high degree of heterozygosity in tea cultivars has hindered the correlation of selected loci with improvement and domestication related traits.

Ancient tea plants grew naturally for hundreds of years without any human cultivation, and the genetic diversity of these plants is important for studying the origin, spread, and classification of tea plants. The ancient tea plants were mainly distributed in the Yunnan-Guizhou Plateau. In this study, we resequenced (more than 10x) a large set of plants representative of the various morphotypes of ancient tea plants from the Yunnan-Guizhou Plateau. Phylogenetic relationships and population structure were assessed and GWAS was performed. RTqPCR was used to verify the expression pattern of genes mined using GWAS in representative ancient tea plants from eight ancient tea populations. Our findings provide useful information for future breeding and molecular identification of tea plants.

\section{Results}

\section{Sequencing and variant discovery}

A total of 120 ancient tea plants from eight groups, including six groups containing 90 individuals from Guizhou Province and two groups containing 30 individuals from Yunnan Province, China, were evaluated in the present study. The geographic distributions of these plants are Xishui (XS), Pu'an (PA), Yanhe (YH), Shiqian (SQ), Duyun (DY), and Sandu (SD) in Guizhou and Lincang (DL) and Menghai (HK) in Yunnan. Detailed information on the agronomic characteristics of the 120 ancient tea plants was obtained based on Chen's study (Fig. 1a, b and Tables S1 and S2) ${ }^{19}$.

Resequencing of the 120 ancient tea plants using the Illumina HiSeq 2000 sequencing platform produced over 5.013 billion raw 150-bp paired-end reads, resulting in $5.01 \mathrm{~Tb}$ of clean data with an average coverage depth of more than 10x (Table S3). Our resequenced reads were mapped onto the published $C$. sinensis var. sinensis genome $^{18,20,21}$. A total of 411,990,204 single nucleotide polymorphisms (SNPs, Fig. 1c) and 18,880,978 indels (insertions and deletions, range $1-54 \mathrm{bp}$, mean $6.6 \mathrm{bp}$, Fig. 1d) were identified. Of the 8,082,370 filtered SNPs (coverage depth $\geq 10$, MAF $<0.05$, and miss rate $\leq 0.1$ ), $1,404,774$ and 175,824 SNPs were distributed in noncoding and coding sequences, respectively. Moreover, 90,502 nonsynonymous SNPs (nsSNPs) were identified in 19,793 genes, and 10,596 frameshift indels were identified in 26,943 genes (Fig. 1c, d). The 6300 variants had a large effect, including SNPs causing premature stop codons or longer-than-usual transcripts and indels resulting in frameshifts, the introduction of stop codons, or other disruptions to protein-coding sequences. To evaluate the selective constraints on ancient tea plants in their natural habitat, the ratio of nonsynonymous to synonymous SNPs $(\mathrm{dN} / \mathrm{dS})$ was calculated and found to be 1.05 . In addition, among all identified SNPs, $2.1 \%$ were located in coding regions: $1.12 \%$ were nonsynonymous and $0.98 \%$ were synonymous (Table S4). This result showed that the proportion of nsSNPs in the coding regions of ancient tea plants was significantly lower than that detected in pear (7.7\%), apple (10.5\%), and soybean (1.9\%), suggesting that less genetic variation occurs in the coding regions of ancient tea plants than in that of fruit trees and some annual crops ${ }^{22-24}$. Moreover, the accuracy of SNP genotyping in randomly selected genomic regions containing a single SNP site was assessed by PCR and Sanger sequencing, revealing an SNP genotype accuracy as high as $98.1 \%$.

\section{Phylogenetic analysis and population structure of ancient tea plants}

To explore the phylogenetic relationships among the 120 ancient tea plants, a phylogenetic tree was constructed by the neighbor-joining (NJ) method using $8,082,370$ SNPs. According to the phylogenetic relationships, the 120 ancient tea plants were mainly clustered into three groups (I-III) and five single branches (Fig. 2a). Among them, group I contained all the members of XS, $\mathrm{DL}, \mathrm{SD}$, and PA and three members of $\mathrm{YH}$; group II contained all the members of HK; and group III contained members of DY, SQ, and $\mathrm{YH}$ and was located close to the cultivated tea plant in the phylogenetic tree (Table S5). This finding is consistent with those of the previous stu$\operatorname{dies}^{25}$. Changes in population structure were further assessed under different $K$ values (Fig. 2b). Analysis of cross-validation error ( $\mathrm{CV}$ error) revealed that seven populations $(K=7)$ represented the best model for these 120 individual ancient tea plants, while the value of $\mathrm{CV}$ error changed little as $K$ increased from 2 to 7 . At $K=2$, the HK members were separated from the main groups. The population structure at $K=3$ was consistent with the three clustered groups in the phylogenetic tree. The XS and DL members were clustered together away from the main groups at $K=4$, and at $K=5$ and 6 , the DL and 


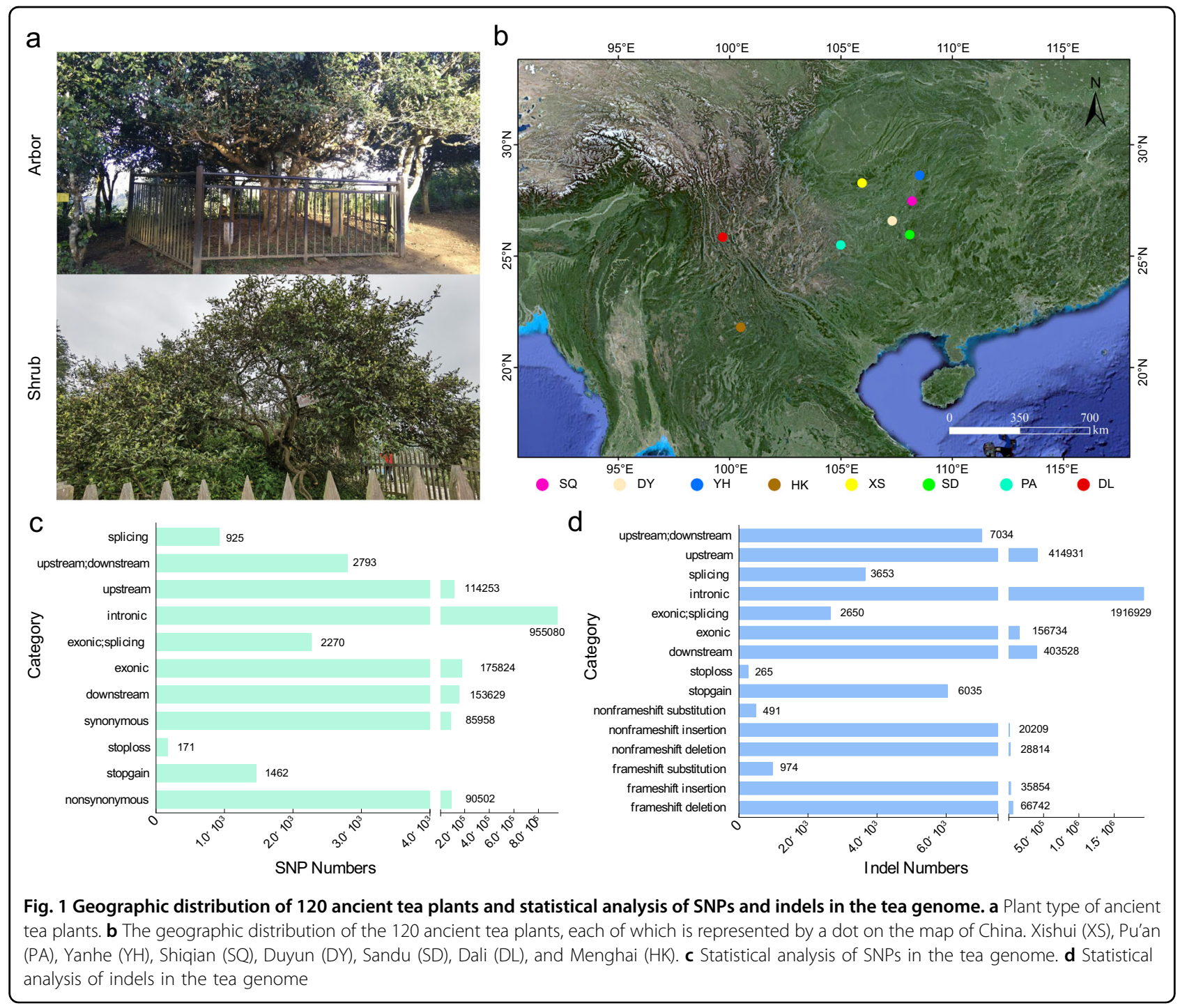

some of the $\mathrm{YH}$ members were clearly separated from the main groups. These results are not only consistent with the geographic distribution of ancient tea plants but are supported by the phylogenetic analysis, indicating that the species in different subgroups (DY, SQ, YH, HK, XS, SD, $\mathrm{PA}$, and DL) from relatively close areas had common geographic origins and that species from the different geographic areas developed independently. The changes in the population structure among the 120 ancient tea plants were mainly related to members of XS, SD, PA, and DL. To identify potential population stratification, principal component analysis (PCA) was used to explore relationships among the 120 ancient tea plants using $\sim 8 \mathrm{M}$ SNPs (Fig. 2c and Fig. S1). PCA revealed three major clusters corresponding to clusters $1-3$ from the phylogenetic tree, which further verifies the accuracy of the phylogenetic tree grouping (Fig. 2a, d). For instance, the PA, SD, XS, and DL samples were clustered together to form cluster 1 , the HK samples were clustered together to form cluster 2, and the $\mathrm{YH}, \mathrm{SQ}$, and DY samples were clustered together to form cluster 3.

\section{Population divergence among ancient tea plants}

Our phylogenetic analysis revealed that genetically close relatives may have similar geographic origins (Fig. 2a, b). Moreover, ancient tea plants from the same place showed similar agronomic traits. For example, most ancient tea plants in DY and SQ were shrub-type plants, whereas those in SD, DL, PA, XS, HK, and YH were tree-type plants. Most ancient tea plants in SQ had dark green leaves, while members of the other groups had light green leaves, and some ancient tea trees in PA had purple leaves. Therefore, some traits and their controlling genes underwent natural screening during the process of geographic isolation. The pairwise population divergence (fixation index: FST) across the PA, DL, DY, and SQ 


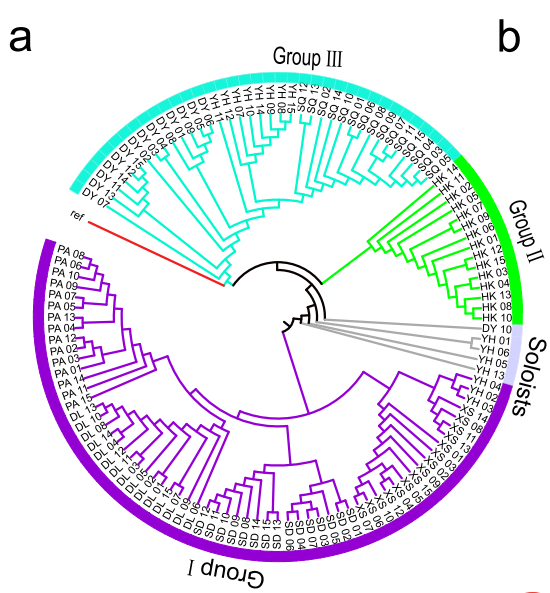

b

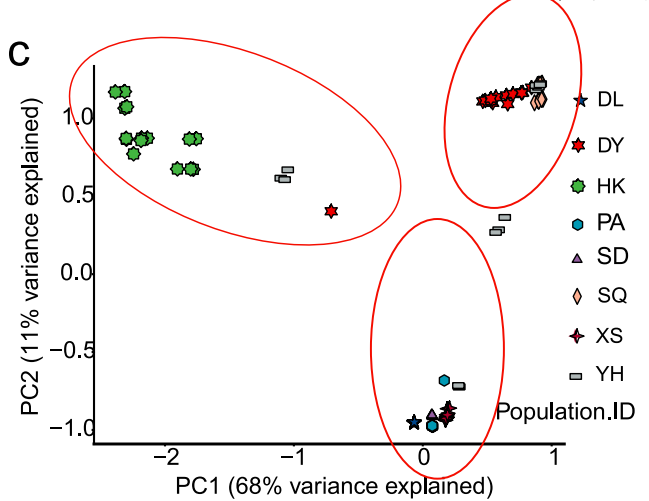

d

\begin{tabular}{llllllllll}
\hline Group & Clade & DL & PA & SD & XS & HK & DY & YH & SQ \\
\hline Group I (Purple) & 1 & 15 & - & - & - & - & - & - & - \\
& 2 & - & 15 & - & - & - & - & - & - \\
& 3 & - & - & 15 & - & - & - & - & - \\
& 4 & - & - & - & 15 & - & - & 3 & - \\
Group II (Green) & 5 & - & - & - & - & 15 & 1 & 3 & - \\
Group III (Blue) & 6 & - & - & - & - & - & 14 & - & - \\
& 7 & - & - & - & - & - & - & 9 & 15 \\
\hline
\end{tabular}

Fig. 2 Population genetic structure of $\mathbf{1 2 0}$ ancient tea plant samples. a NJ tree of 120 ancient tea plants inferred from SNPs at fourfold degenerate sites. A phylogenetic tree was constructed using MEGA software with default parameters. b Model-based Bayesian clustering of 120 ancient tea plants performed using ADMIXTURE Version 1.3.0 with the number of ancestry kinships (K) set to 2-7. Each accession is denoted by a vertical bar composed of different colors corresponding to its proportion of genetic ancestry from each of these populations. $\mathbf{c}$ Principal component analysis of the 120 ancient tea plants. $\mathbf{d}$ The geographic origin of each accession in the seven clades

subgroups was analyzed (Fig. 3a, c). The mean FST among the ancient tea plants in DL and DY was 0.745, suggesting obvious population divergence, which was followed by a mean FST of 0.217 among the plants in DL and HK. However, the mean FST among the ancient tea plants in DY and SQ was 0.08, suggesting little population divergence.

Tajima's D was used to evaluate whether the observed nucleotide diversities showed evidence of deviation from neutrality. Some regions were significantly different from zero, indicating natural or artificial selection (Fig. 3b). Of these, the D values from the SD, DL, PA, DY, XS, HK, YH, and SQ genomes were mostly positive, indicating a predominance of intermediate-frequency SNPs in these subgenomes. The genome-wide nucleotide diversity $(Ө \pi)$ across all ancient tea plants was $6.1 \times 10^{-3}$. This value was higher than that of other perennial crops, such as peach $\left(1.5 \times 10^{-3}\right)$, cassava $\left(2.6 \times 10^{-3}\right)$, and pear $\left(5.5 \times 10^{-3}\right)$, but lower than that reported for date palm $\left(9.2 \times 10^{-3}\right)^{26-28}$. The genetic diversity decreased from $6.1 \times 10^{-3}$ in ancient tea plants to $5.15 \times 10^{-5}$ in improved tea cultivars ${ }^{29}$, suggesting the loss of significant genetic diversity during domestication.

\section{Genome-wide association studies}

Linkage disequilibrium (LD indicated by $r^{2}$ ) analysis indicated that the ancient tea plant genome has a relatively short $r^{2}$ distance and rapid $r^{2}$ decay (Fig. 4b). The $r^{2}$ decreased to half its maximum value, at $19.3 \mathrm{~kb}$, which is higher than the $r^{2}$ in cultivated tea plants $(5 \mathrm{~kb})$ but lower than that in ancient tea plants $(\sim 40 \mathrm{~kb})$ reported by $\mathrm{Xia}^{9}$. Moreover, the ancient tea plants from DL showed the highest $r^{2}$ value $\left(r^{2}=40.0 \mathrm{~kb}\right)$, and those from DY showed the lowest $r^{2}$ value $\left(r^{2}=11.6 \mathrm{~kb}\right)$ (Fig. S2). The LD decay distance for the ancient tea plants was much longer than that for pear (211 bp) and apple (161 bp) but much shorter than that for soybean $(150 \mathrm{~kb})$ and rice $(123 \mathrm{~kb})$.

Plant domestication conducted over several millennia has resulted in the modification of specific plant traits, including leaf size, shape, texture, width, color, the 

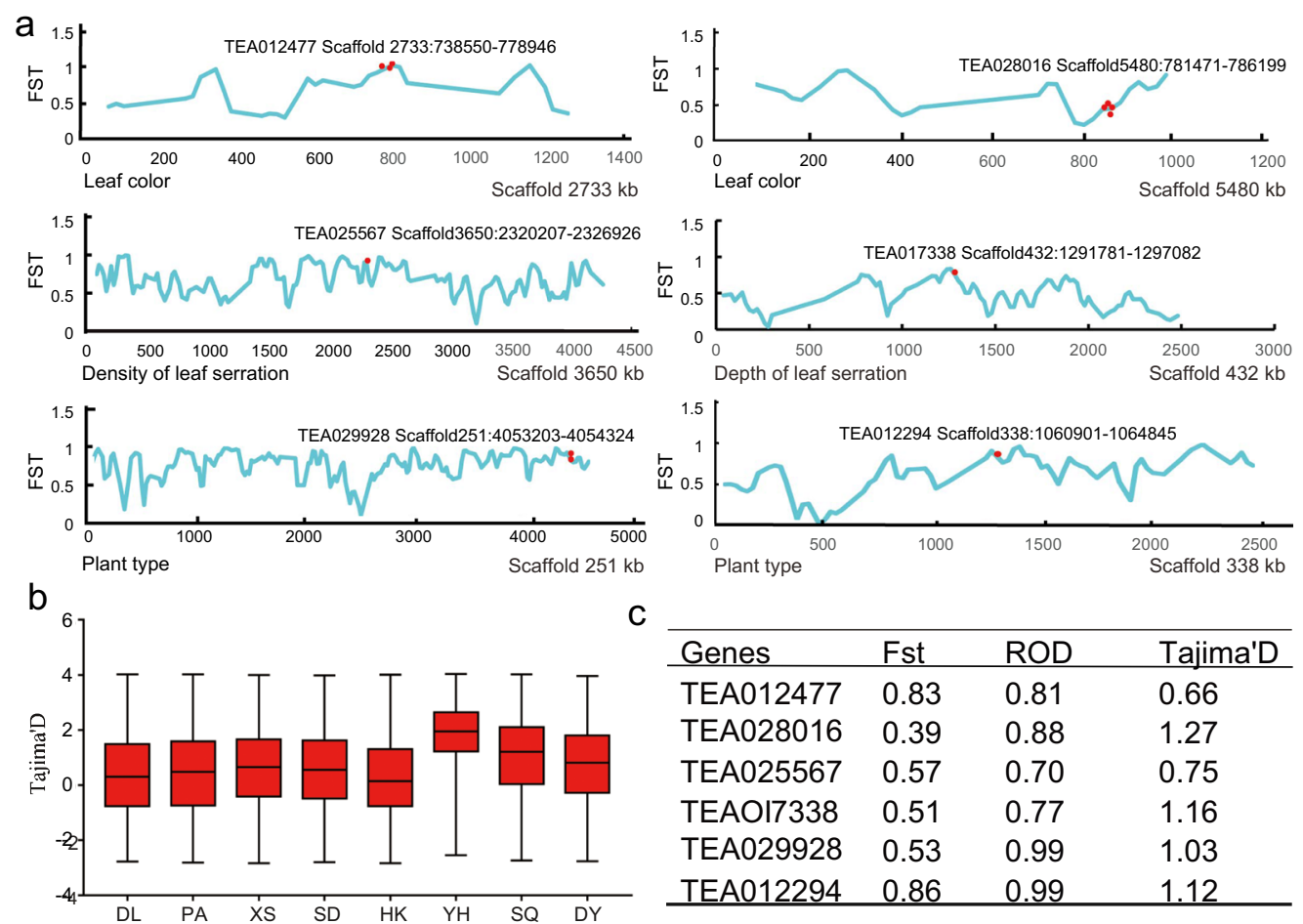

C

\begin{tabular}{llll}
\hline Genes & Fst & ROD & Tajima'D \\
\hline TEA012477 & 0.83 & 0.81 & 0.66 \\
TEA028016 & 0.39 & 0.88 & 1.27 \\
TEA025567 & 0.57 & 0.70 & 0.75 \\
TEAOI7338 & 0.51 & 0.77 & 1.16 \\
TEA029928 & 0.53 & 0.99 & 1.03 \\
TEA012294 & 0.86 & 0.99 & 1.12 \\
\hline
\end{tabular}

Fig. 3 Population sweeps during local adaptation among ancient tea plants from the different areas. a The distribution of FST along the scaffold. $\mathbf{b}$ The distribution of Tajima's D along the scaffold. $\mathbf{c}$ Selection signals estimated by the three measures for seven candidate genes for leaf and plant type

number of leaf veins, and the density and depth of leaf serration $^{19,30}$. To further screen the candidate genes associated with eleven leaf traits, compression multilocus random mixed linear model analysis was conducted for GWAS using GAPIT software (Fig. 4a). To obtain highquality SNPs, imputation was performed for the ancient tea plant SNP set, retaining 8,082,370 SNPs with a MAF of $5 \%$. In total, 1176 SNPs were associated with 11 target leaf traits, and 292 loci were involved in regulating plant type. Most of the loci that were associated with ancient leaf traits and plant type are shown here for the first time (Figs. S3-S7 and Table S6).

\section{Candidate genes involved in the regulation of leaf traits}

In tea plants, the leaves are rich in characteristic compounds, such as polyphenols, caffeine, theanine, vitamins, polysaccharides, volatile oils, and minerals. Leaf inclusions are often related to many leaf traits, including leaf size, color, and the number of leaf veins. GWAS signals associated with leaf traits were detected in the present study (Fig. 4c, d and Table 1). Three nsSNPs were identified to have associations with leaf color: two nsSNPs in TEA012477 and one nsSNP in TEA028016 $\left(-\log _{10} P \geq\right.$ 8.2). Functional annotation inferred that these two genes encode calmodulin-binding transcription activator 2 and the Cop1/SPA ubiquitin ligase complex, respectively. The latter is involved in the repression of anthocyanin accumulation under low- and high-light conditions in Arabidopsis (Table 1). An nsSNP was identified to be significantly $\left(-\log _{10} P \geq 8.2\right)$ related to the density of leaf serration and caused a change from $A$ to $C$ at base 428 in the CDS of TEA025567, resulting in a change from Glu to Ala at residue 143. Moreover, an nsSNP was found to be significantly $\left(-\log _{10} P \geq 8.2\right)$ related to the depth of leaf serration and caused a change from $\mathrm{C}$ to $\mathrm{T}$ at base 320 in the CDS of TEA017338, resulting in a change from Ser to Phe at residue 107. In addition, the ancient tea plants were classified into shrub and arbor plants. Two nsSNPs were also identified to be significantly associated with plant type and caused a change from A to T at base 322 and from $\mathrm{G}$ to A at base 532 in the CDS of TEA029928, resulting in changes from Thr to Ser at residue 108 and from Gly to Ser at residue 178, respectively. Moreover, an nsSNP (G/A) was found at base 476 in the CDS of TEA01294, resulting in a change from Gly to Glu at residue 159. Annotation and functional analysis of homologous genes in Arabidopsis showed that these two genes encode an F-box protein and an acyl carrier protein. In Arabidopsis, F-box proteins repress ethylene action and promote growth by directing EIN3 degradation (ethylene restricts Arabidopsis growth via the epidermis), and acyl 

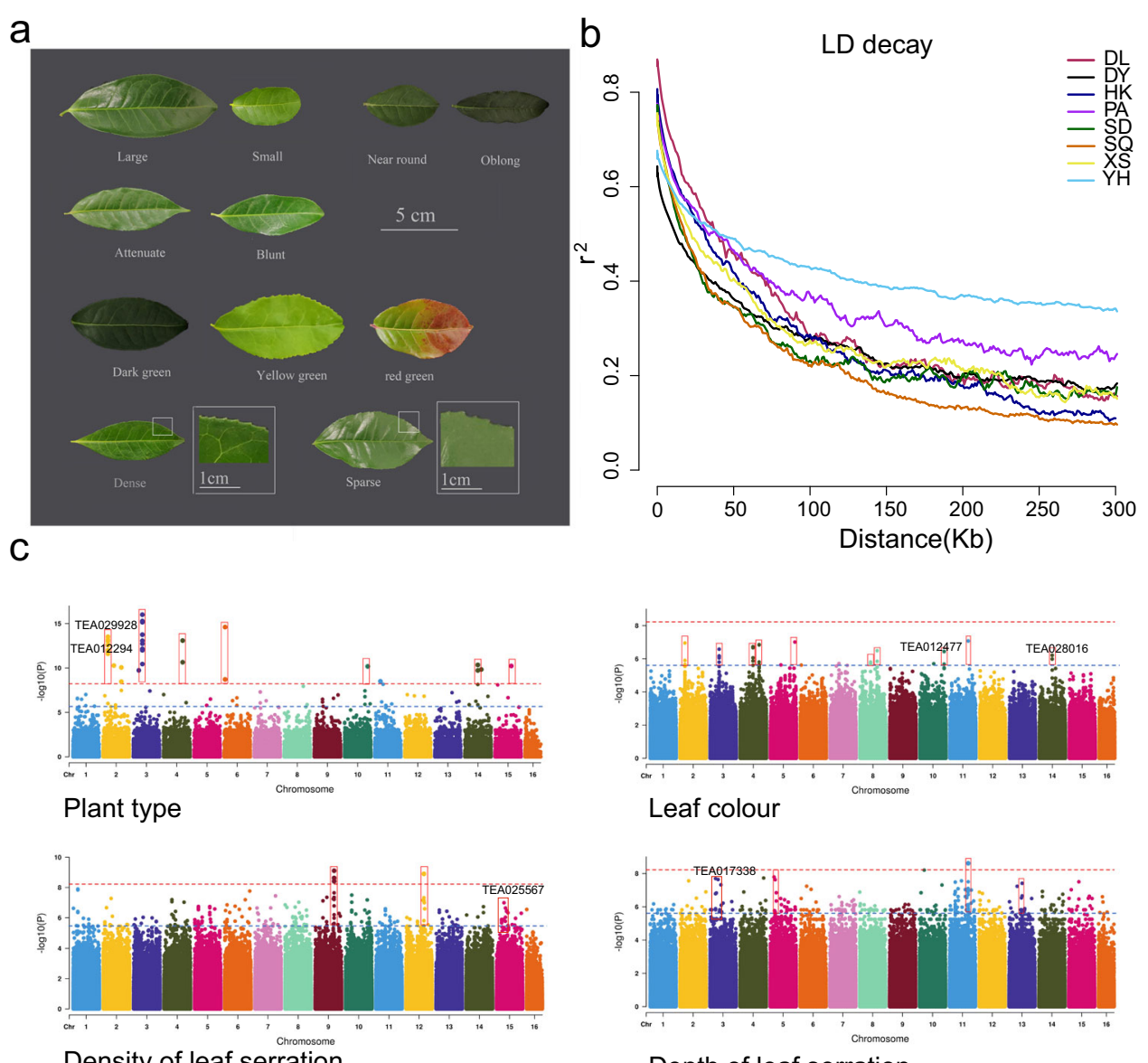

Density of leaf serration

Depth of leaf serration

d

TEA012294
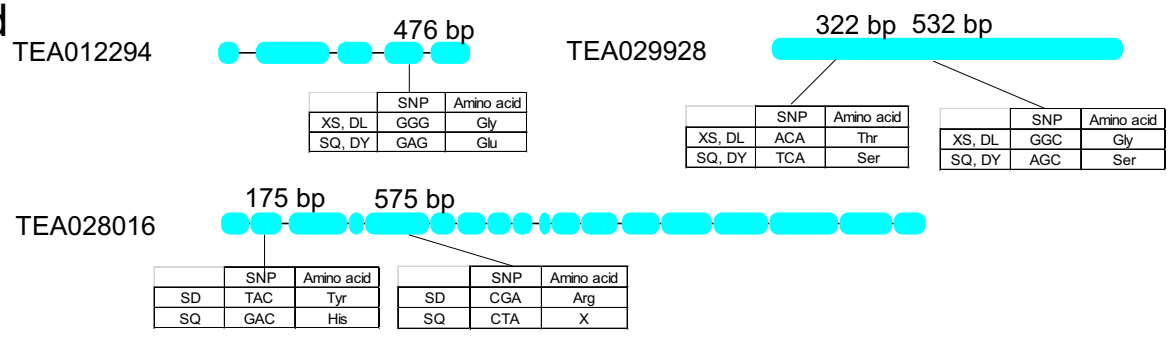

TEA012477
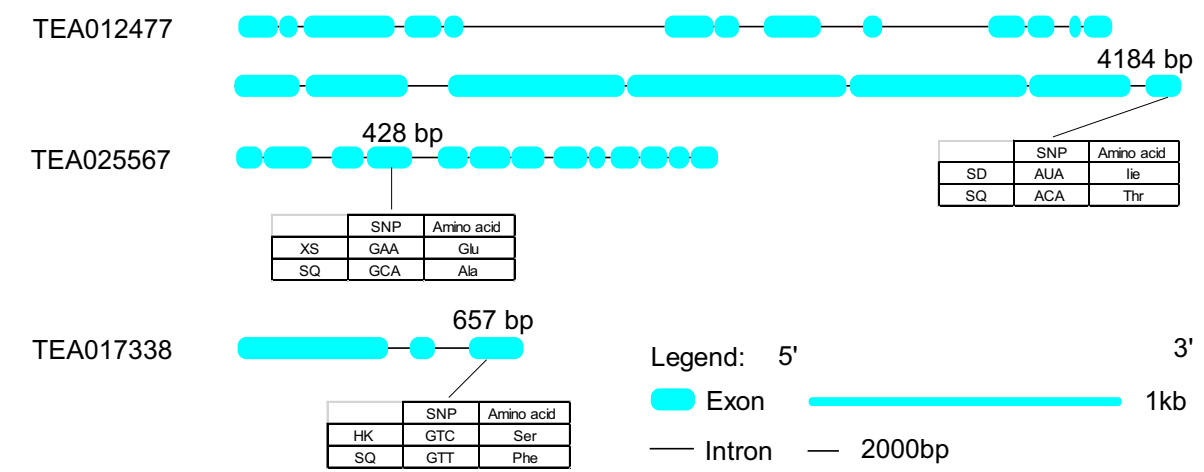

Fig. 4 Regions related to leaf traits in the ancient tea plants. a Leaf traits of the 120 ancient tea plants. $\mathbf{b} L D$ analysis of the 120 ancient tea plants. c Manhattan plots for four traits. The significance threshold of the $-\log _{10} P$ value was set at 5.5 (blue). $\mathbf{d}$ Structure of genes related to leaf traits according to GWAS 
Table 1 GWAS results for genes associated with different traits

\begin{tabular}{|c|c|c|c|c|c|c|}
\hline Trait & Gene locus & Exon & SNP sites & Protein & $-\log _{10}(P)$ & Gene annotation \\
\hline \multirow[t]{5}{*}{ Leaf color } & TEA012477 & 15 & CGT (1826)-GAT & $\operatorname{Arg}(609)-H i s$ & 1.16718 & Calmodulin-binding transcription activator 2 \\
\hline & & 18 & TCC (3406)-GCC & Ser (1136)-Ala & 0.93987 & \\
\hline & & 20 & ATA (4184)-ACA & Ile (1394)-Thr & 8.43856 & \\
\hline & TEA028016 & 2 & TAC (175)-GAC & Tyr (59)-His & 6.21486 & Cop1 ubiquitin ligase complex \\
\hline & & 5 & CGA (574)-CTA & Arg (192)-Leu & 6.21486 & \\
\hline Density of leaf serration & TEA025567 & 4 & GAA (428)-GCA & Glu (143)-Ala & 8.90128 & Tetratricopeptide repeat (TPR) protein \\
\hline Depth of leaf serration & TEA017338 & 3 & GTC (657)-GTT & Ser (107)-Phe & 7.32429 & AT-rich interactive domain protein \\
\hline \multirow[t]{2}{*}{ Plant type } & TEA029928 & 1 & GGC (532)-AGT & Gly (178)-Ser & 8.08721 & F-box protein \\
\hline & TEA012294 & 1 & CTG (475)-TGT & Gly (159)-Glu & 8.46766 & Acyl carrier protein 2 \\
\hline
\end{tabular}

carrier proteins control plant architecture by regulating the cytokinin signaling pathway.

To further verify the differences among the different populations, we selected two genes (TEA012477 and TEA029928) related to leaf color and plant type to detect the distribution of nsSNPs in representative plants of eight different populations. As shown in Fig. S8, we isolated and aligned the homologous sequences of the TEA029928 and TEA012477 genes from eight representative individuals from eight populations. One nsSNP was identified to be significantly $\left(-\log _{10} P \geq 8.2\right)$ related to plant type, causing a change from a $\mathrm{C}$ in arbor-type plants (DL, HK, XS, PA, SD, and $\mathrm{YH}$ ) to an A in shrub-type plants (SQ, DY, and $\mathrm{YH})$ at base 532 in the CDS of TEA029928, resulting in a change from Glu to Ser at residue 178. Moreover, three nsSNPs were significantly $\left(-\log _{10} P \geq 8.2\right)$ related to leaf color, causing changes from G, G, and C in light green plants (DL, HK, XS, PA, SD, $\mathrm{YH}$, and $\mathrm{DY}$ ) to $\mathrm{A}, \mathrm{T}$, and $\mathrm{T}$ in dark green plants (SQ) at bases 1826, 3406, and 4184 in the CDS of TEA012477, resulting in a change from Arg, Ala, and Thr to His, Ser and Ile at residues 609, 1136, and 1394.

To further explore the functional differences among different groups, RT-qPCR was used to investigate the expression level of the four representative genes mined using GWAS among different populations with different traits. Our results showed that the expression level of the gene TEA021477 in ancient tea trees with light green leaves was significantly lower than that in ancient tea trees with dark green leaves. The expression level of the gene (TEA029928) related to plant type in shrub-type ancient tea plants was significantly higher than that in arbor-type ancient tea plants (Fig. S9). In addition, a previous study revealed that the density and depth of leaf serration were quantitative traits controlled by multiple genes. Dynamic expression changes in the genes related to leaf serration of different densities and depths were investigated using RT-
qPCR, suggesting that these two traits were controlled by multiple genes ${ }^{31}$. The nsSNPs were used as molecular markers to distinguish the difference between shrub- and arbor-type ancient tea plants, which were also used to determine the difference between light green and dark green leaves (Tables S7, S8 and Fig. S9).

\section{Discussion}

Although C. sinensis "Fuding Dabaicha" has been widely planted in the southwestern region of China due to its high yield and economic value, there remain many ancient tea plant resources that have not been exploited ${ }^{8}$. In the present study, we generated a dataset encompassing the considerable genomic variation of ancient tea plants, which provided an opportunity to explore the divergence, population structure, and regulatory mechanisms of related traits in ancient tea plants. A previous study suggested that tea plants have diverse origins and that Chinese cultivated tea plants originated from southwestern China and later spread to western $\mathrm{Asia}^{28}$. The results of our phylogenetic analysis show that the 120 ancient tea plants were mainly clustered into three groups and five single branches. Three members of $\mathrm{YH}$ were clustered together with the ancient tea plants from XS and distributed in group 1, indicating that gene exchange occurred between them, which could be partially attributed to the consistent introgression among the ancient tea plants during the long cultivation process 9 . Our results further reveal that the ancient tea plants in DL and PA are more ancient than those in the other six populations, and the ancient tea plants in DY and SQ are closely related to the cultivated tea plant based on phylogenetic analysis, which is consistent with the findings of the previous studies ${ }^{28}$. It has been demonstrated that ancient tea plants from DY are closer to modern cultivars ${ }^{32}$. Based on the phylogenetic analysis, the ancient tea plants from DY and SQ were a sister branch to the cultivars, which is consistent with the 
results of a previous study. The ancient tea plants of XS clustered outside of the clade containing the SD, DL, and PA groups, suggesting that the members of XS are more ancient than the members of the SD, DL, and PA groups. Moreover, some members of $\mathrm{YH}$ clustered outside the clade containing the HK, DY, and SQ groups, suggesting that some members of $\mathrm{YH}$ are more ancient than the members of the HK, DY, and SQ groups.

The results of LD analysis demonstrated that natural selection pressures acted during the evolution and domestication of ancient tea plants. The ancient tea plants in DL showed the highest $\mathrm{LD}\left(r^{2}=467 \mathrm{~kb}\right)$, indicating that these plants experienced the greatest selection pressure. The results of kinship $(K)$ analysis further revealed that the 120 ancient tea plants were clustered into seven subgroups with monophyletic clades from the same or nearby places, indicating a common geographic origin.

Artificial breeding of cultivated tea plants dramatically reduces genetic diversity ${ }^{9}$. As a precious natural resource, ancient tea plants have higher genetic diversity, which is of great value for studying the evolutionary mechanism and diversification of tea plants. This phenomenon is consistent with results in crops such as rice ${ }^{33}$. As expected, a number of outlier regions were identified, and 19 candidate genes were found to contain 107 SNPs associated with plant type and leaf traits, including leaf length, width, size, shape, texture, color, the number of leaf veins, and the density and depth of leaf serration. Among these genes, six genes with eight SNPs were significantly associated with four traits based on KEGG annotation and functional analysis of orthologous genes in Arabidopsis, suggesting that these candidate genes screened by GWAS may be involved in regulating the development of leafrelated traits and plant type (Table 1). In Arabidopsis, the Cop1/SPA ubiquitin ligase complex is involved in repressing anthocyanin accumulation under low- and high-light conditions and the F-Box protein (corresponding to the functional gene of TEA029928) regulates leaf size ${ }^{34,35}$. Moreover, the soybean stearoyl-acyl carrier protein (corresponding to the functional gene of TEA023604) regulates the different morphological phenotypes of the leaves ${ }^{36,37}$. Furthermore, NADH dehydrogenase (ubiquinone) 1 alpha (corresponding to the functional gene of TEA012294) regulates the different morphologies of the plant (Table 1$)^{38,39}$.

Our study provides a valuable resource for understanding the phylogenetic relationships, population structure, and genetic diversity of ancient tea plants in southwestern China. In addition, candidate genes significantly associated with four important agronomic traits were identified. The significant SNPs associated with favorable variants, selection signals, and candidate genes are a valuable resource for the further improvement of leaf traits and plant type in ancient tea plants.

\section{Materials and methods}

Plant material and agronomic evaluation

A total of 120 ancient tea plants were selected to represent a broad distribution of geography, morphology, and genetic diversity. These plant materials included 90 individual ancient tea plants from Guizhou, China, and 30 individual ancient tea plants from Yunnan, China, which were classified and standardized according to the book of Chinese tea plants ${ }^{19}$. The different values represent the different qualitative and quantitative traits based on the classification and standardization of tea plants.

\section{Sequencing, mapping, and SNP calling}

Total DNA was extracted using the modified cetyltrimethylammonium bromide (CTAB) method ${ }^{40}$. At least $6 \mu \mathrm{g}$ of genomic DNA from each individual was used to construct a sequencing library following the manufacturer's instructions (Illumina Inc.). Paired-end sequencing libraries with an insert size of $\sim 400$ bp were sequenced on an Illumina HiSeq 2000 sequencer at BGI company. The draft genome sequence of the tea plant $(C$. sinensis var. sinensis $\mathrm{cv}$. shuchazao), downloaded from the TPIA database (http://tpia.teaplant.org/), was used as a reference genome ${ }^{9,41}$. Paired-end reads were mapped to the tea reference genome with BWA (v 0.6.1) software using the default parameters ${ }^{42}$. SAMtools software was used to convert mapping results into the BAM format and to filter the unmapped and nonunique reads ${ }^{43}$. The Picard package was used to filter the duplicated reads ${ }^{42}$. The CoverageBed program in BEDtools v2.17.0 was used to calculate the coverage of sequence alignments ${ }^{44}$. After alignment, SNP calling was conducted per individual using SAMtools ${ }^{45}$. The genotype likelihoods were evaluated from the reads of each individual at each genomic location. A Bayesian approach was used to determine the allele frequencies. SNPs were identified by the samtools mpileup command. To remove false positives, only $8,082,370$ high-quality filtered SNPs (coverage depth $\geq 10$, MAF $<0.05$, and miss rate $\leq 0.1$ ) were used in the subsequent analysis.

\section{Functional annotation of genetic variants}

SNP annotation was conducted based on the draft genome of $C$. sinensis var. sinensis using the ANNOVAR package ${ }^{9,46,47}$. According to the annotation information, SNPs were distributed in exonic regions, splicing sites, $5^{\prime}$ UTRs, 3' UTRs, intronic regions, upstream and downstream regions (which were distributed in $1 \mathrm{~kb}$ regions away from the transcription start site), and intergenic regions. Moreover, SNPs in exonic regions were further divided into synonymous SNPs (sSNPs) or nsSNPs. Indels in the coding regions were identified based on frame-shift mutations (3 bp insertion or deletion). 


\section{Phylogenetic tree and population genetics analysis}

To explore the phylogenetic relationship of ancient tea plants at the genome-wide level, an NJ tree was constructed using the p-distance in MEGA v7.0 software with bootstrap values determined from 1000 replicates ${ }^{48}$.

Admixture software was used to analyze the population structure of 120 ancient tea plants with $K$ values ranging from 2 to $13^{28}$. PCA was also used to evaluate the genetic structure of the ancient tea populations using GCTA software $^{49}$.

\section{Linkage disequilibrium analysis}

To compare the patterns of LD among the different ancient tea populations, the squared correlation coefficient $\left(r^{2}\right)$ between pairwise SNPs was computed using PopLDDecay 3.26 software with default parameters ${ }^{50}$. The average $r^{2}$ value was calculated for pairwise markers in a $100-\mathrm{kb}$ window and averaged across the whole genome.

\section{Genome-wide association study}

The $\mathrm{R}$ package GENESIS v.2.14.157 was employed to perform GWAS between genotypes and phenotypes for all quantitative and qualitative data ${ }^{51}$. The genetic relationship matrix (GRM) can be used as a generalized linear mixed model of random effects to explain population stratification. In the present study, it was used to test the abovementioned association. GCTA v.1.92.158 was applied to calculate the $\mathrm{GRM}^{52}$. The count data adopted a Poisson distribution, and the remaining quantitative data adopted a Gaussian distribution. If necessary, a Box-Cox power transformation was used, and normality was verified using a Shapiro-Wilk test. For qualitative traits, a binomial distribution was assumed, while for multiple qualitative traits, each category level was treated as a virtual binary variable. Quantile-quantile plots were used to evaluate the GWAS model (Figs. S4-S8 a, b). The GEC (Genetic Type 1 Error Calculator) v. 0.259 was used to estimate the significance level of correlation ${ }^{53}$. Compression multilocus random mixed linear model analysis was conducted for GWAS using GAPIT software ${ }^{54}$. The GWAS correction threshold was $8.2\left(-\log _{10}(0.05 /\right.$ 8082370)) (Manhattan plot, red dotted line).

Population fixation statistics (FST) and reduction of diversity (ROD) were calculated for nonoverlapping genomic intervals in 1-kb windows using VCFtools ${ }^{55}$. All the output results of ROD and FST were standardized and transformed into $z$-scores using a 100 -kb sliding window with a 10-kb step size. The outlier windows of ROD and FST with high values were used to identify candidate genes based on $z$-tests with a significance level of $\alpha=0.05$ corresponding to a $z$-score of 1.645 . The population genetics statistic Tajima's D was calculated directly from short-read alignments using ANGSD with nonoverlapping 10-kb intervals (version 0.609$)^{56}$.

\section{Extraction of RNA and RT-qPCR analysis}

The total RNA of ancient tea plants was isolated using a Huayueyang Plant RNA Extraction Kit (Quick RNA isolation Kit; Haidian District, Beijing). The expression patterns of four genes identified by GWAS were measured using RT-qPCR. RT-qPCR was performed using SYBR Premix Ex Tag (TaKaRa) using cDNA as the template. The results were analyzed using the $-\Delta \Delta C T$ method with GAPDH gene expression as an internal reference. Three biological and three technical replicates were used ${ }^{57}$.

\section{Acknowledgements}

We thank Guizhou University for providing a platform for our experiments and BGl for performing sequencing and analysis for this project. This work was supported by the Technology Creation Center of Guizhou Tea Industrialization (Qiankezhongyindi [2017]4005); Guizhou Tea Industrial System-Function Laboratory of Tea Nutrition and Cultivation [K20-68-006]; and Research on Key Technologies of the Quality Improvement of White, Yellow, and Purple Varieties (Qiankehe Platform Talent [2019]5651); Screening and evaluation of tea germplasm resources with high EGCG in Guizhou based on SSR molecular marker technology (Qiankehe LH word [2017] No. 7269).

\section{Author details}

${ }^{1}$ College of Tea Science, Guizhou University, Guiyang 550025, People's Republic of China. ${ }^{2}$ College of Life Sciences and The Key Laboratory of Plant Resources Conservation and Germplasm Innovation in the Mountainous Region (Ministry of Education), Institute of Agro-Bioengineering, Guizhou University, Guiyang 550025, People's Republic of China. ${ }^{3}$ Guizhou Academy of Agricultural Sciences, Guiyang 550025, People's Republic of China

\section{Author contributions}

L.L. and D.Z. designed the experiments; H.C. and X.W. performed the experiments and data analyses; B.X. participated in data analyses; H.C., X.W., and X.Y. wrote the manuscript; L.L., D.Z., X.W., H.C., X.Y., B.X., and Y.D. revised the manuscript. All authors read and approved the final manuscript.

\section{Data availability}

The datasets presented in this study can be found in online repositories. The names of the repositories and accession numbers can be found below: PRJNA716079 (Table S9).

\section{Conflict of interest}

The authors declare no competing interests.

Supplementary information The online version contains supplementary material available at https://doi.org/10.1038/s41438-021-00617-9.

Received: 2 September 2020 Revised: 20 May 2021 Accepted: 24 May 2021 Published online: 10 August 2021

\footnotetext{
References

1. Willson, K. C. \& Clifford, M. N. Tea: cultivation to consumption. Ecol. Freshw. Fish. 5. 175-182 (1992).

2. Mondal, T. K., Bhattacharya, A., Laxmikumaran, M. \& Ahuja, P. S. Recent advances of tea (Camellia sinensis) biotechnology. Plant Cell Tiss. Org. 76, 195-254 (2004).

3. Yamamoto, T., Juneja, L. R., Chu, S. \& Kim, M. Chemistry and Applications of Green Tea (CRC Press, 1997).

4. Cabrera, C., Artacho, R. \& Giménez, R. Beneficial effects of green tea-a review. J. Am. Coll. Nutr. 25, 79-99 (2006).

5. Rogers, P. J., Smith, J. E., Heatherley, S. V. \& Pleydell-Pearce, C. W. Time for tea: mood, blood pressure and cognitive performance effects of caffeine and theanine administered alone and together. Psychopharmacology 195 569-577 (2008).
} 
6. Chacko, S. M., Thambi, P. T., Kuttan, R. \& Nishigaki, I. Beneficial effects of green tea: a literature review. Chin. Med. 5, 13 (2010).

7. Hayat, K., Iqbal, H., Malik, U., Bilal, U. \& Mushtaq, S. Tea and its consumption: benefits and risks. Crit. Rev. Food Sci. 55, 939-954 (2013).

8. Xia, E. H. et al. The tea tree genome provides insights into tea flavor and independent evolution of caffeine biosynthesis. Mol. Plant. 10, 866-877 (2017)

9. Xia, E. H. et al. Tea plant information archive: a comprehensive genomics and bioinformatics platform for tea plant. Plant Biotech. J. 17, 1938-1953 (2019).

10. Hufford, M. B. et al. Comparative population genomics of maize domestication and improvement. Nat. Genet. 44, 808-811 (2012).

11. Jiao, Y. P. et al. Genome-wide genetic changes during modern breeding of maize. Nat. Genet. 44, 812-815 (2012).

12. Qi, J. J. et al. A genomic variation map provides insights into the genetic basis of cucumber domestication and diversity. Nat. Genet. 45, 1510-1515 (2013).

13. Levy, D. et al. Genome-wide association study of blood pressure and hypertension. Nat. Genet. 41, 677-687 (2009).

14. Su, J. et al. Genome-wide association study identified genetic variations and candidate genes for plant architecture component traits in Chinese upland cotton. Theor. Appl. Genet. 131, 1299-1314 (2018).

15. Wang, M. et al. Genome-wide association study (GWAS) of resistance to head smut in maize. Plant Sci. 196, 125-131 (2012).

16. Zhang, J. P. et al. Genome-wide association study for flowering time, maturity dates and plant height in early maturing soybean (Glycine max) germplasm. BMC Genomics 16, 217-228 (2015).

17. $\mathrm{Wu}$, J. et al. Genome-wide association study (GWAS) of mesocotyl elongation based on re-sequencing approach in rice. BMC Plant Biol. 15, 1-10 (2015).

18. Wei, C. L. et al. Draft genome sequence of Camellia sinensis var. sinensis provides insights into the evolution of the tea genome and tea quality. Proc. Natl Acad. Sci. USA 115, 4151-4158 (2018)

19. Chen, L., Yang, Y. J., Yu, F. L., Yao, M. Z. \& Wang, X. C. Descriptors and data standard for tea (Camellia spp.). China Agriculture Press 1, 3-6 (2005).

20. Wang, W. S. et al. Genomic variation in 3,010 diverse accessions of Asian cultivated rice. Nature 557, 43-49 (2018).

21. $W u$, J. et al. Diversification and independent domestication of Asian and European pears. Genome Biol. 19, 1-16 (2018).

22. Duan, N. B. et al. Genome re-sequencing reveals the history of apple and supports a two-stage model for fruit enlargement. Nat. Commun. 8, 1-11 (2017).

23. Lam, H. M. et al. Resequencing of 31 wild and cultivated soybean genomes identifies patterns of genetic diversity and selection. Nat. Genet. 42, 1053-1062 (2010).

24. Cao, K. et al. Comparative population genomics reveals the domestication history of the peach Prunus persica and human influences on perennial fruit crops. Genome Biol. 15, 415-430 (2014)

25. Zhang, X. Q. et al. Diversity analysis of agronomic and quality traits of tea plant resources in Guiding Bird King. Mol. Plant Breed. 13, 415-423 (2015)

26. Wang, W. et al. Cassava genome from a wild ancestor to cultivated varieties. Nat. Commun. 5, 1-9 (2014).

27. Hazzouri, K. M. et al. Whole genome re-sequencing of date palms yields insights into diversification of a fruit tree crop. Nat. Commun. 6, 1-11 (2015)

28. Ge, J. X. in China's Belt and Road Initiatives (ed. Liu, W.) 10-14 (Springer, 2018).

29. Xia, E. H. et al. The reference genome of tea plant and resequencing of 81 diverse accessions provide insights into genome evolution and adaptation of tea plants. Mol. Plant. 13, 1013-1026 (2020).

30. $\mathrm{Xu}, \mathrm{X}$. et al. Resequencing 50 accessions of cultivated and wildrice yields markers for identifying agronomically important genes. Nat. Biotechnol. 30, 105-111 (2011).

31. Krisztina, N. et al. The balance between the MIR164A and CUC2 genes controls leaf margin serration in Arabidopsis. Plant Cell 18, 2929-2945 (2006).
32. Zhang, X. Q., Zhou, F. Y., Yang, C., Zhang, Z. Q. \& Hu, J. Q. Diversity of tea germplasm resource (Camellia sinensis 'Guiding-niaowangzhong') revealed based on agronomic and quality traits. Mol. Plant Breed. 13, 415-423 (2015).

33. Yano, K. et al. Genome-wide association study using whole-genome sequencing rapidly identifies new genes influencing agronomic traits in rice. Nat. Gene. 48, 927-934 (2016).

34. Woo, H. R. et al. ORE9, an F-Box protein that regulates leaf senescence in Arabidopsis. Plant Cell 13, 1779-1790 (2001).

35. Joke, B. et al. F-Box protein FBX92 affects leaf size in Arabidopsis thaliana. Plant Cell Physiol. 5, 962-975 (2017).

36. Lakhssassi, N. et al. Stearoyl-acyl carrier protein desaturase mutations uncover an impact of stearic acid in leaf and nodule structure. Plant Physiol. 174 1531-1543 (2017).

37. Gou, L. et al. Multigene synergism increases the isoflavone and proanthocyanidin contents of Medicago truncatula. Plant Biotech. J. 14, 915-925 (2016)

38. Gagne, J. M. et al. Arabidopsis EIN3-binding F-box 1 and 2 form ubiquitinprotein ligases that repress ethylene action and promote growth by directing EIN3 degradation. Proc. Natl Acad. Sci. USA 101, 6803-6808 (2013).

39. Takato, S. et al. Auxin signaling through SCFIR1/AFBs mediates feedback regulation of IAA biosynthesis. Biosci. Biotech. Biochem. 81, 1-7 (2017).

40. Murray, M. \& Thompson, W. F. Rapid isolation of high molecular weight plant DNA. Nucleic Acids Res. 8, 4321-4326 (1980).

41. Li, H. \& Durbin, R. Fast and accurate short read alignment with BurrowsWheeler transform. Bioinformatics 25, 1754-1760 (2009).

42. $\mathrm{Li}, \mathrm{H}$. et al. The sequence alignment/map format and SAMtools. Bioinformatics 25, 2078-2079 (2009).

43. Kim, J. E., Oh, S. K, Lee, J. H. \& Jo, S. H. Genome-wide SNP calling using next generation sequencing data in tomato. Mol. Cells 37, 36-42 (2014).

44. Quinlan, A. R. \& Hall, I. M. BEDTools: a flexible suite of utilities for comparing genomic features. Bioinformatics 26, 841-842 (2010).

45. Megan, J. et al. Supercomputing for the parallelization of whole genome analysis. Bioinformatics 30, 1508-1513 (2014).

46. Josh, C. et al. Single nucleotide polymorphism identification in polyploids: a review, example, and recommendations. Mol. Plant 8, 831-846 (2015).

47. McKenna, A. et al. The genome analysis toolkit: a MapReduce framework for analyzing next-generation DNA sequencing data. Genome 20, 1297-1303 (2010).

48. Wang, K., Li, M. \& Hakonarson, H. ANNOVAR: functional annotation of genetic variants from high-throughput sequencing data. Nucleic Acids Res. 38, 164 (2010).

49. Sudhir, K., Glen, S. \& Koichiro, T. MEGA7: molecular evolutionary genetics analysis version 7.0 for bigger datasets. Mol. Biol. Evol. 7, 1870-1874 (2016).

50. Yang, J., Lee, S. H., Goddard, M. E. \& Visscher, P. M. GCTA: a tool for genomewide complex trait analysis. Am. J. Hum. Genet. 88, 76-82 (2011).

51. Stephanie, M. G. et al. Genetic association testing using the GENESIS R/Bioconductor package. Bioinformatics 35, 5346-5348 (2019).

52. Zhang, C., Dong, S. Shan, Xu, J. Y., He, W. M. \& Yang, T. L. PopLDdecay: a fast and effective tool for linkage disequilibrium decay analysis based on variant call format files. Bioinformatics 35, 1786-1788 (2018).

53. Browning, B. L. \& Browning, S. R. Genotype imputation with millions of reference samples. Am. J. Hum. Genet. 98, 116-126 (2016).

54. Lipka, A. E. et al. GAPIT: genome association and prediction integrated tool. Bioinformatics 15, 2397-2399 (2012).

55. Danecek, P. et al. The variant call format and VCFtools. Bioinformatics 27 2156-2158 (2011)

56. Durvasula, A. et al. ANGSD-wrapper: utilities for analyzing next generation sequencing data. Mol. Ecol. Resour. 16, 1449-1454 (2016).

57. Wang, X. J. et al. Characterization and expression analysis of ERF genes in Fragaria vesca suggest different divergences of tandem ERF duplicates. Front. Genet. 10, 1-13 (2019). 